\title{
Zero Suicide: A Big Hairy Audacious Goal
}

Joe Rafferty

Mr. Joe Rafferty is one of the co-founders of the Zero Suicide Alliance, a national suicide prevention collaboration of over 1500 members across the UK and the globe. Before coming to Mersey Care, which hosts the ZSA, Joe held the post of Director of Commissioning Support at the NHS Commissioning Board, having national responsibility for the design and delivery of a significant component of the commissioning side reforms outlined in the White Paper: Liberating the NHS. Prior to this, he was seconded from the Chief Executive role at NHS Central Lancashire, to become the Director of Commissioning Development for NHS North West, with a remit to lead the development of commissioning reforms across the North West of England. From 2007 to 2010 he was the Chief Executive of NHS Central Lancashire and the Regional Director of Commissioning and Strategy for NHS North West from 2006-2008. Other Board-level roles have included Director of Performance in Cumbria and Lancashire SHA and Director of System Reform at Bolton Hospital NHS Trust. Prior to these roles, he was part of the team that set up Greater Manchester SHA.

Abstract. This presentation explores the benefits of using audacious, zerobased thinking to stimulate change in healthcare provider and wider societal thinking in relation to suicide prevention. The Zero Suicide Alliance (ZSA) was born out of one mental health trust's thrust to begin to radically re-engineer it's thinking and delivery in relation to suicides of patients in their care. This approach of zero suicide created a focus and release of clinical creativity that has fundamentally alter the organisation's position on suicide prevention, it's approach to service redesign and its overall philosophy on learning - giving rise to a comprehensive and successful Just Culture programme. These benefits have become the centre point for establishing the Zero Suicide Alliance, a bottom up movement in the UK with a philosophy of knowledge into action. The presentation explores the benefits of the zero suicide approach and describes the work of the ZSA. 